\title{
Application of Virtual Reality Technology in Library
}

\author{
Chunxu Guan a, Yujiao Liang \\ Gannan Medical University, Jiangxi Ganzhou 341000, China \\ a775101514@qq.com
}

Keywords: virtual reality technology; library; document retrieval.

\begin{abstract}
Virtual reality technology has three characteristics of immersion, interactivity and imagination, and has added new method of multi-dimensional touch and human-computer interaction, which can be widely applied in library construction, information resource construction and reader service. Therefore, the library should actively pay attention to mutually use virtual reality technology and two image generation technologies, intensify advertisement, and promote virtual reality technology.
\end{abstract}

\section{Introduction}

With rapid development of modern information technology, the capability of information processing has been greatly improved. It is not only required to observe information processing results through printing output, screen display and such methods, but also hoped to be in the information processing environment through vision, auditory, touch and body, gesture or command to have am immersive experience. Library, as intelligence information center, has been directly influenced by the rapidly-developing information technology, and has been unprecedentedly changed in terms of reader serving method, technology method, service contents and others. The traditional library mode has suffered from new challenge, and it develops towards digitization and virtualization. Virtual reality technology has brought brand new experience to library, which fully improves the application of information technology in library.

\section{Virtual Reality Technology}

\subsection{Virtual reality technology}

Virtual reality is a comprehensive integration technology, involved with computer graphics, sensor technology, human-computer interaction techniques, artificial intelligence and other fields. With computer technology as core, it combines with other scientific technologies to generate lifelike three-dimensional vision, auditory sense, smell, touch and other senses, enabling users to experience and interact with the virtual world in a virtual environment by relevant equipment and devices. Computer system can promptly conduct complex calculation with movement of users positions, transmitting accurate images of the virtual 3D world to make users feel like being in a real world. This technology integrates with and reserves the latest developed achievements of modern computer graphics technology, sensor technology, artificial intelligence, display technology, parallel internet processing technology and other technologies, generating lifelike high-tech analog system by computer technology.

\subsection{Basic characteristics of virtual reality technology}

Virtual reality technology has four basic characteristics, including immersion, imagination, interactivity and multi-sensory. Immersion, also called as telepresence, refers to the realness degree felt by users when being in an analog environment as the leading player. Imagination focuses on emphasizing that there is wider imagination space for application of virtual reality technology, which can not only re-present real existing environment, but also image the not-existing and even impossible virtual environments. Interactivity refers to the operational degree of objects in the analog environment and nature degree of getting feedback. Multi-sensory refers to that virtual reality 
technology has multi-sensory capabilities, such as visual perception, auditory perception, tactile perception and even the more advanced taste perception, olfactory perception, etc.

\section{Application of Virtual Reality Technology in Library}

How to provide readers with individual and personal services has become an important target for library development and reform. Library takes important measures to improve reader service, including paying attention to science and technology development, and actively using high technology to serve the users. There are still shortcomings existing in traditional library working procedure, such as resources flattening, outdated reader service working method, etc. The virtual reality technology, as a human-computer interface based on nature, emphasizes on human-orientation to make people feel like being in the real environment. Therefore, it is necessary to promote virtual reality technology in library industry. With development and improvement of computer software and hardware, immersion virtual reality system will be absolutely as popular as computer.

\subsection{Scene construction}

Scene construction is the visual generation part of virtual reality technology, namely to generate a lifelike virtual scene. To design a virtual library scene with immersion with existing library as prototype is the primary problem for application of virtual reality technology in library. It can not only realize to wander in the National Library Phase II project, but also proceed specific works such as books shelve arrangement, shelves adjustment, selection of reading room positions, etc, by establishing virtual library scene. Display with virtual image can make librarian work more convenient, and save manpower and material resources consumed by physical adjustment and design.

\subsection{Information resource construction}

Information resource construction refers to library collection construction, namely that using virtual reality technology to change the existing information resources which include paper resource and data resource. Adopt virtual reality technology to display knowledge in books in virtual image based on digital library collection to maximize the education function of library. What's adopted in the existing state is three-dimensional information resource which can effectively change flattening of the existing information resource, and provide three-dimensional resources for readers. Three-dimensional of information resource refers to conducting virtual reality technology processing to physical carrier of information resource, so that it can enable readers to fell like reading books in real library. E.g. The "virtual reality reading station" in the National Library Phase II Project adopts Flash technology and human body gesture identification system to provide browser service and interaction experience of digital library for readers in the north area of the National Library, where readers can read electronic books on computer by the non-contact virtual interaction to realize virtual reading of e-books. Current, the provided contents include documents of the Republic of China, award of article ferry books, local chronicles and famous ancient books. This new book reading method can not only avoid damage to original ancient books due to humanistic reasons, but also provide readers with a feeling of reading physical books, which greatly increases reader's interest.

\subsection{Readers development}

As entering knowledge economy era, and with deepening of life-long learning concept, people have been paying increasing attention to improve self knowledge and quality, urgently needing various kinds of knowledge and information, which provides an opportunity for development of library, one of the institutes preserving and passing human culture. However, approaches for human beings to obtain information knowledge have become diversified. Therefore, it becomes an important problem for library to attract more readers to keep its position in information culture communication. Virtual reality technology can change library's image of dull and cold book collection room in users' mind, such as three dimensional display method of information resource and virtual environment of existing library buildings, which all add fun to reading, and will attract more citizens to enter library to use information resources in library. For users in and not in library, the immersion and interactivity of virtual reality technology are exactly identical to reading psychology of existing readers, for reading can not only expand readers' knowledge, but also be an entertainment behavior. The 
successful case of the National Library applying virtual reality technology fully shows that virtual reality technology plays a role of vital importance in attracting readers. Virtual reality technology emphasizes natural interaction between people and virtual environment to enable users to obtain their necessary knowledge by simple methods, which will bring a brand new library image to readers.

\subsection{Document retrieval}

Traditional document retrieval procedure is: when readers need to borrow books, they firstly inquire the circulation status and call number of the book, and then go to the open-shelf book stack room to get the book according to the call number. There are physical book getting problems in this traditional retrieval method. It would usually take long time for readers to find the shelf where the book is in and the specific position of the book after entering the book stack room and find the book according to the call number, and sometimes readers can not find the book in the stack room since other readers did not put their borrowed books in original positions or librarian did not put the returned books in shelf. Virtual reality technology can display the entire library building and three-dimensional position of each book in the three-dimensional environment in details through the three-dimensional modeling of the library. Readers can firstly find out the specific position of their necessary book, and it can generate an optimal guidance path at the same time, namely from the current position to the shelf of the corresponding book.

\subsection{Education and training to users}

It usually teaches knowledge related to information retrieval by opening courses in school, holding training class and lectures, and other method in previous library user education and training. In these methods, generally readers should to participate in the library, which greatly limits training scale. American educationalist, Edgar Dale comes up with "Experience Tower" theory in his Visual \& Audio Teaching Method, and divided the learned experience into three categories in ten levels according to abstraction degree: doing experience (including direct experience with goals, design experience, and playing experience); observing experience (including observation and demonstration, field trip, visiting, television and file, static image, broadcast and recording); abstract experience (including visual sing and language sing). During library users education and training, adopt virtual reality technology to build an immersion virtual environment where learners can devote themselves into learning by extremely natural method and environment and real-time interaction with other learners or teachers, which is much more helpful to obtain doing and observing experience. Virtual reality technology can provide virtual training scene for readers needing to receive training to make readers feel like learning in real environment, which can not save costs for readers to learn in library, but also increase training quality and expand training scale.

\section{Conclusion}

With increasingly improved social emphasis on library, expenditure input in library has been increasingly growing, especially for support to digital library, and library has to take measures to actively correspond to resource and service transition under network information environment. Application of virtual reality technology makes library service, service contents and service scope more diversified, which can truly display library building characteristics, reading environment and library collection resources, and simulate to wander in library space to enable readers to fully and directly understand the library and virtually read data resources collected in the library by gesture, sensory and other non-contact methods. When obtaining sensory experience and learning in entertainment, readers can reinforce their understanding to the library, and be led to make the most of resources of the library.

\section{References}

[1] Wang Shuo.Application of 3D Virtual Browsing Technology in Digital Library Construction Based on Virtools_-3D Books Navigation System of Capital Normal University Library[J].New Technology of Library and Information Service,2011(Z1):121-126. 
[2] Zhang Puyang,Ding Hongyan,Bai Zhihua.Virtual Reality and Visual Simulation Technology in Platform Construction with Four Bucket Foundations Based on Secondary Development of Vega[J]. Journal of tianjin university (English edition),2013,19(6):398-403.

[3] Alcinia Z. Sampaio,Octavio P. Martins.The application of virtual reality technology in the construction of bridge: The cantilever and incremental launching methods[J].Automation in construction,2014,37(Jan.):58-67. 